\title{
An openwork like structures of polylactide - manufacturing and properties
}

\author{
Karolina Sobczyk ${ }^{1, *}$, and Karol Leluk ${ }^{1}$ \\ ${ }^{1}$ Wroclaw University of Science and Technology, Faculty of Environmental Engineering, \\ Wybrzeze Wyspianskiego 27, 50-370 Wroclaw, Poland
}

\begin{abstract}
Poly(lactic acid) electrospinning tests were carried out under various process conditions. Openwork structures with a high surface area to weight ratio have been obtained. Changing the parameters of the PLA electrospinning process resulted in products with different fiber morphology.
\end{abstract}

\section{Introduction}

Electrospinning is a process patented by A. Formhals, who was first to describe the production of cellulose acetate fibers in the early years of twentieth century [1]. Electrospinning, an electrostatic fiber fabrication technique has evinced more interest and attention in recent years due to its versatility and potential for applications in diverse fields. The notable applications include tissue engineering, biosensors, filtration, wound dressings, drug delivery, and enzyme immobilization [2]. The interest in fabrication of nanofibers using electrospinning method has attracted considerable attention due to its versatile maneuverability of producing controlled fiber structures, porosity, orientations and dimensions [3].

Poly(lactic acid) (PLA) is a bio-based biodegradable polymer that can be produced from renewable resources including starch from corn and potatoes, sugar from beets and sugar cane, and so forth. The carbon in PLA originates from atmospheric carbon dioxide, which is immobilized in glucose by photosynthesis; therefore, the carbon dioxide formed by its disposal, incineration, or biodegradation does not increase the total amount of atmospheric carbon dioxide. Poly(lactic acid) and its copolymers have attracted significant attention in environmental, biomedical, and pharmaceutical applications and as alternatives to petrobased polymers [4]. The fibers obtained by the electrospinning method have found a wide application, inter alia, in medicine for the production of antiseptic dressings and as scaffolds for tissue engineering [5] and as a filter material due to the possibility of obtaining porous structures [6]. The interest in fabrication of nanofibers using electrospinning method has attracted considerable attention due to its versatile maneuverability of producing controlled fiber structures, porosity, orientations and dimensions. Although the process appears to be simple and straightforward, an understanding of the technique and its influence on the morphology, structural

\footnotetext{
* Corresponding author: karolina.sobczyk.pwr@gmail.com
} 
and mechanical properties is still not completely clear [3]. Polymer fibers can be obtained by several techniques, including drawing, phase separation and electrospinning from solution. The diameter of fibers obtained as a result of this process is in the range of: $100 \mathrm{~nm}-1 \mu \mathrm{m}$ and the length of a single fiber can reach several kilometers [7].

The electrospinning process includes understanding electrostatics, fluid rheology, and polymer solution properties, such as solvent evaporation rate, its surface tension and conductivity. These basic properties constantly affect each other during the electrospinning process. Also the product of the process - is influenced by a large number of factors. Generally, they have been classified in three main groups:
a) equipment parameters: voltage applied, solution flow rate, collector type, distance between nozzle tip and collector, needle diameter.
b) parameters of the solution: viscosity, electrical conductivity, dielectric permittivity, surface tension.
c) ambient parameters: temperature, humidity.

\section{Materials and methods}

The aim of the conducted research was to obtain nanofibres with a developed specific surface area. For this purpose, a series of polylactide polymer solutions (PLA) was prepared in a chloroform $\left(\mathrm{CHCl}_{3}\right)$. The surface morphology of the obtained fibers was determined by scanning electron microscopy (SEM). Materials chemical characteristics was performed using FT-IR investigation.

\subsection{Characterization of reagents}

Poly lactic acid (PLA) solutions were prepared in chloroform, manufactured by "CHEMPUR" company with mean molecular mass $119.38 \mathrm{~g} / \mathrm{mol}$ and purity $99.5 \%$. Polylactic acid used during tests is a granulate available under the name of IngeoTM Biopolimer 3052D manufactured by NatureWorks. Its most signifiacant properties are listed in the Table 1.

Table 1. IngeoTM Biopolimer 3052D physical parameters.

\begin{tabular}{|c|c|}
\hline Parameter & Value \\
\hline Melting temperature, ${ }^{\circ} \mathrm{C}$ & 200 \\
\hline Moisture content, $\%$ & $<0.025$ \\
\hline Tensile Elongation, $\%$ & 3.5 \\
\hline MFR $\left(2.16 \mathrm{~kg}, 210^{\circ} \mathrm{C}\right), \mathrm{g} / 10 \mathrm{~min}$ & 14 \\
\hline
\end{tabular}

\subsection{Sample preparation}

The tests were carried out on a series of solutions differing in concentrations (shown in table below).

Table 2. Concentration of polymer solutions used for the electrospinning process.

\begin{tabular}{|c|c|}
\hline & Concentration of the solution \\
\hline Sample 1 & $9.2 \%$ \\
\hline Sample 2 & $10.6 \%$ \\
\hline Sample 3 & $10.6 \%$ \\
\hline Sample 4 & $11.9 \%$ \\
\hline Sample 5 & $14.5 \%$ \\
\hline Sample 6 & $14.5 \%$ \\
\hline
\end{tabular}


For each sample the preparation procedure was as follows:

1) Weighting of desired PLA quantity

2) Measured volume of solvent $\left(40 \mathrm{ml} \mathrm{CHCl}_{3}\right)$.

3) The prepared mixture was left on a magnetic stirrer to accelerate the dissolution of the granulate at room temperature.

4) The prepared clear solution was placed in a syringe that was part of the Fluidnatek LE-10 system.

\subsection{Research system}

The tests were carried out on the FLUIDNATEK LE-10 equipment from the spanish company Bioinicia. It consists of three main parts:

- $\quad$ infusion pumps with syringe and nozzle,

- collector for fiber collection,

- $\quad$ high voltage supply.

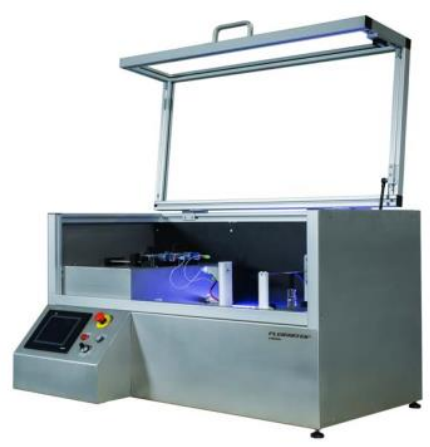

Fig. 1. FLUIDNATEK LE-10.

The morphology of the obtained materials was made on the basis of analysis of images obtained from the scanning electron microscope SEM - VEGA Tescan 3.

\subsection{Adjustment of parameters}

During the tests, a number of changes in process parameters were divided into three main groups of factors affecting the electrospinning process:

a) Equipment parameters

- The applied voltage value - according to the use of high voltage, the electric charges of the solution and the external electric field start the electrospinning process. The voltage applied is intended to distort the solution at the end of the needle, followed by the formation of the Taylor cone. In most cases, applying a higher voltage leads to more stretched fibers of smaller diameter. This case also leads to faster evaporation of the solvent from the solution [8].

- Solution flow rate - the solution delivery rate in the system is controlled by the pump and the diffusion phenomenon. The rate at which the solution is fed into the system affects the formation of Taylor's cone. Too high speed of the solution may cause dripping from the dispensing nozzle without the formation of a suitably thin thread, too low speed, however, it may lead to thread breakage or clogging of the dosing nozzle [8]. 
- Collector distance from the injector - solvent complete evaporation is crucial for the process - it stimulates thin fibers production. Changing the distance between the tip of the nozzle and the collector will have a direct effect on the flight time as well as the strength of the electric field. In order to produce fibers, the solution stream must have time to evaporate the solvent. When the distance between the tip and the collector is reduced, the flow will have a shorter path before it reaches the collector. The intensity of the electric field and the acceleration of the flow of the stream will increase [8].

- Needle diameter - the smaller the diameter of the nozzle, the more likely it is to have a blockage at the nozzle or at its end. In addition, reducing the internal diameter of the hole may cause a reduction in the diameter of the fibers. It is caused by the increase in the surface tension of drops [9].

b) Solution parameters

- Concentration - depending on the solubility of the polymer, a good solvent allows higher polymer concentration while maintaining a liquid, stable solution, while the inferior solvent will have more polymer-polymer contacts between the different chains and lead to gelation. The effect of the solvent on the intrinsic viscosity also depends on the elasticity of the polymer chain. If the polymer chain is more rigid, the effect of the solvent on the intrinsic viscosity is lower [8].

c) Ambient parameters

- Temperature - temperature has a direct effect on the rate of evaporation of the solution. The higher the temperature, the more solvent evaporates [8].

- Humidity - high humidity can cause pores formation on the surface of fibers. This phenomenon is related to the condensation of water on the surface of the fiber. As a result, it affects the morphology of the fibers, especially in the case of polymers dissolved in volatile solvents. The humidity of the environment also determines the rate of evaporation of the solvent from the solution. At very low humidity the volatile solvent will evaporate faster [8].

\subsection{FT-IR (ATR) measurement}

The infrared spectra was obtained for material before dissolution and after electrospinning process. Investigation was performed in the wavenumber range $600-4000 \mathrm{~cm}^{-1}$ using Bruker Tensor 37 apparatus and Opus software. The samples of electrospun materials were placed in the apparatus without any further processing. PLA pellets (solid material before solving) were grinded. Prior to that, according to the pellets toughness, the materials were immersed in a liquid nitrogen for about 3 minutes. The powdered polymer sample was then placed into the apparatus measuring cell and the spectra was recorded. Baseline correction, normalization, spectrum smoothening and any further necessary calculations (like peak picking) were done within the measuring procedure time-length. No spectra processing was done after the measurement.

\subsection{SEM investigation}

Scanning electron microscope was used to investigate the morphology of the process products. It was carried on VEGA Tescan 3 , gun voltage $5.0 \mathrm{kV}$, magnification rate is mentioned on the respective pictures and text. Samples were gold sputtered (Kressington 108 sputter coater) for $60 \mathrm{~s}$ and $40 \mathrm{~mA}$ current. 


\section{Results and discussion}

For solutions with different concentrations (Table 2) electrospinning process was carried out. The parameters changed during the process are: the distance between the tip of the nozzle and the collector, the applied voltage, the speed of the solution flow, were additionally recorded the temperature and humidity of the air in the chamber of the device. The obtained materials, in order to determine their morphology, were analyzed by scanning electron microscope VEGA Tescan 3. The results of research and analysis of SEM images are presented in Table 3.

Table 3. List of process parameters and SEM images for selected samples.

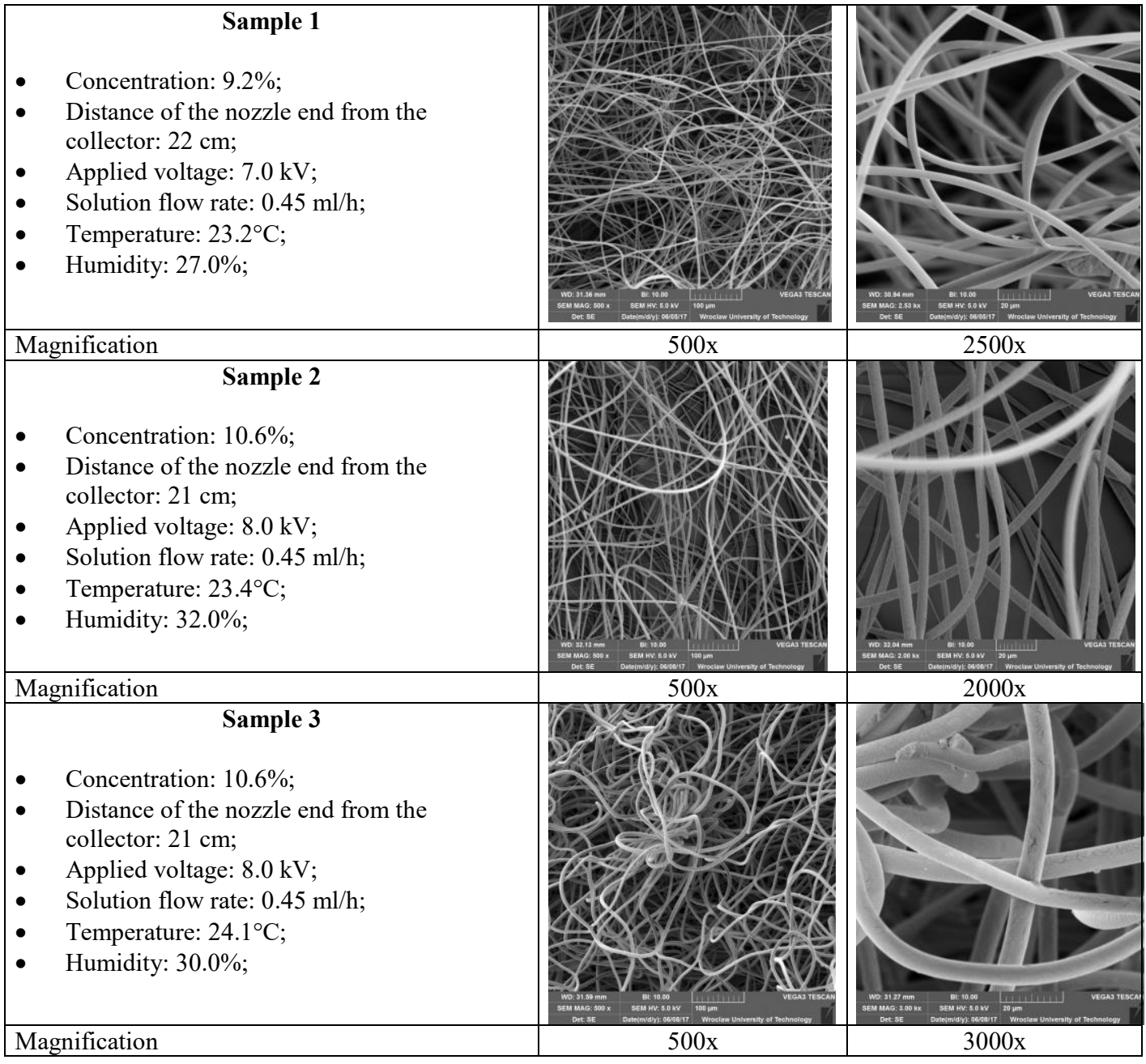




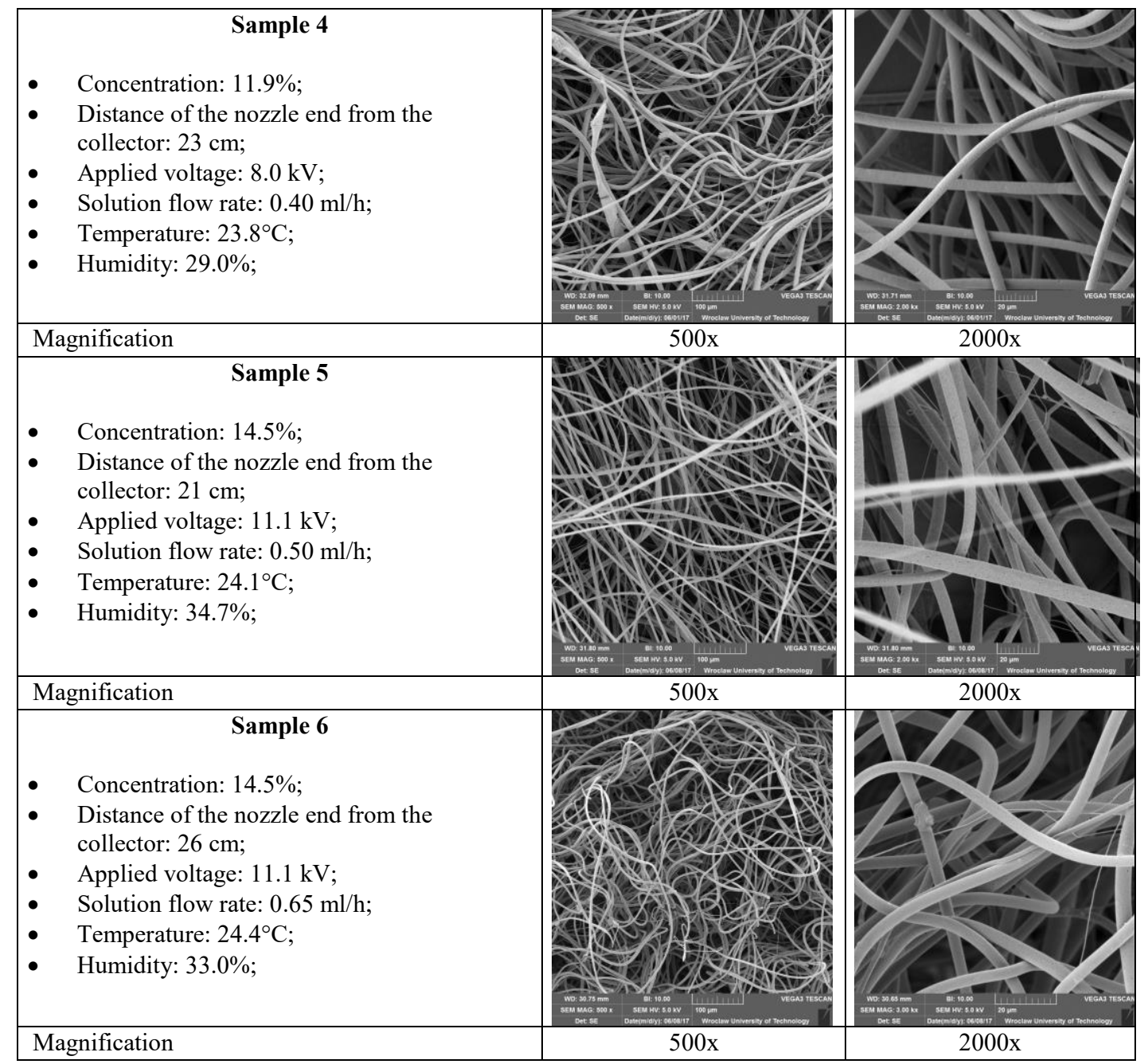

Sample 1 - With the parameters used for the $9.23 \%$ concentration, regular, porous fibers (with a significant number of pores, uniformly shaped and sized pores, evenly distributed over the entire fiber surface) were obtained, cylindrical.

Sample 2, Sample 3 - Regular, non-porous, cylindrical fibers were used with the parameters used for the $10.60 \%$ concentration.

Sample 4 - Regular, non-porous, cylindrical fibers were obtained with the parameters used for the $11.94 \%$ concentration.

Sample 5, Sample 6 - With the parameters used for the concentration of $14.49 \%$ obtained regular, porous (with a significant number of pores, pores of uniform shape and size, evenly distributed over the entire surface of the fibers), cylindrical.

Figure 2 show FT-IR investigation results for unprocessed PLA and electrospun fibers whereas in the table 4 charateristic signals were collected. 


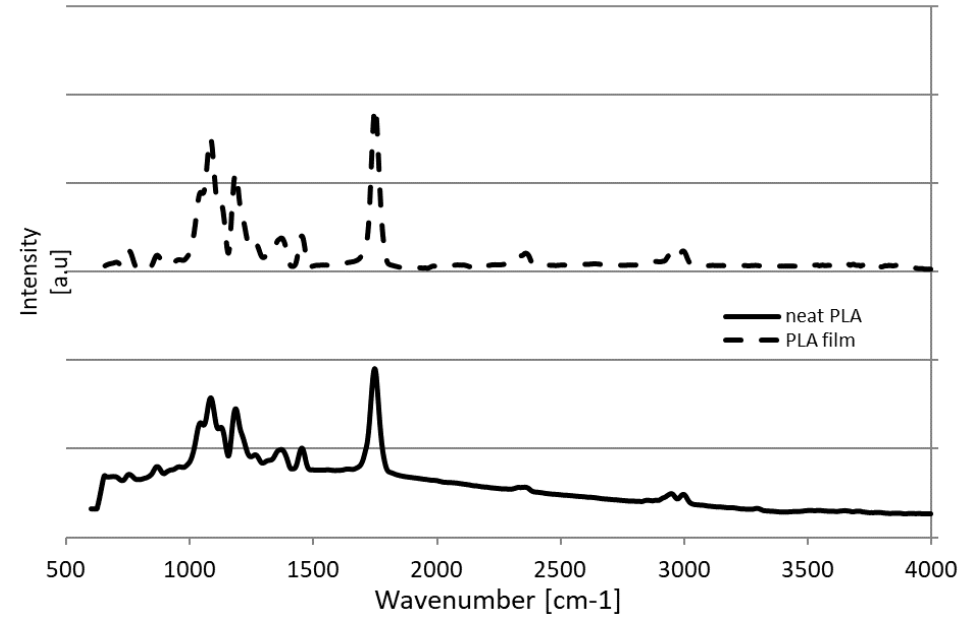

Fig. 2. FT-IR investigation results for neat PLA and PLA electrospun fibers.

Table 4. Characteristic signals for polylactic acid in unprocessed and electrospun fibers.

\begin{tabular}{|c|c|c|c|c|}
\hline System & Wavenumber & Intensity (Abs) & Rel Intensity & Width \\
\hline \multirow{3}{*}{ Neat PLA } & 1747 & 1.9 & 1.4 & 41.2 \\
\cline { 2 - 5 } & 1184 & 1.5 & 0.5 & 39.8 \\
\cline { 2 - 5 } & 1083 & 1.6 & 0.9 & 80.5 \\
\hline \multirow{3}{*}{ PLA film } & 1749 & 1.9 & 1.8 & 37.9 \\
\cline { 2 - 5 } & 1184 & 1.1 & 0.9 & 42.5 \\
\cline { 2 - 5 } & 1082 & 1.5 & 1.5 & 72.7 \\
\hline
\end{tabular}

As it can be clearly seen from above graphical representations, no significant differences may be observed in FT-IR spectra of investigated material.

\section{Conclusions}

- On the basis of conducted tests, possibility to create openwork structures from the PLA polymer solution is possible utilizing electrospinning process;

- Parameters were established for obtaining smooth or porous fibers in the determined solution concentration; by their alternation manufacturing process of desired fibers is easy operation;

- The materials obtained as a result of the tests consist of fibres with different thickness, being a result of a specific collector type (flat) used in the investigation;

- According to FT-IR data no significant changes were observed in PLA structure.

\section{References}

1. E. Tyrolczyk, K. Wielgus, M. Szalata, J. Makowicka, Chemik 66, 1219 (2012)

2. N. Bhardwaj, S.C. Kundu, Biotechnol. Adv. 28, 325 (2010)

3. A. Baji, Y.W. Mai, S.C. Wong, M. Abtahi, P. Chen, Compos. Sci. Technol. 70, 703 (2010) 
4. S. Kabasci, H. Tsuji, Bio-Based Plastics: Materials and Applications (John Wiley \& Sons, Ltd., 2013)

5. S. Agarwal, J.H. Wendorff, A. Greiner, Polymer 49, 5603 (2008)

6. M. Kwiatkowska, M. Kozłowski, Polimery-W 60, 7 (2015)

7. J. Michna, S. Irusta, A. Kryzioł, Zeszyty Naukowe Towarzystwa Doktorantów UJ - Nauki Ścisłe 11, 7 (2015)

8. S. Ramakrishna, K. Fujihara, W. Teo, T. Lim, Z. Ma, An introduction to electrospinning and Nanofibers (World Scientific Publishing Company, Singapore, 2005)

9. A. Baranowska-Korczyc, Dissertation: Pótprzewodnikowe sensory oparte na nanowtóknach otrzymywanych metoda elektroprzedzenia (Institute of Physics of the Polish Academy of Sciences, Warsaw, 2012) 